\title{
Do patients with Parkinson's disease with freezing of gait respond differently than those without to treadmill training augmented by virtual reality?
}

Esther MJ Bekkers, $\mathrm{PhD}^{1}$, Anat Mirelman, $\mathrm{PhD}^{2,3}$, Lisa Alcock, $\mathrm{PhD}^{4}$, Lynn Rochester, $\mathrm{PhD}^{4}$, Freek Nieuwhof, $\mathrm{PhD}^{5}$, Bastiaan R Bloem, $\mathrm{PhD}, \mathrm{MD}^{5}$, Elisa Pelosin, $\mathrm{PhD}^{6,7}$, Laura Avanzino, $\mathrm{PhD}, \mathrm{MD}^{7,8}$, Andrea Cereatti, $\mathrm{PhD}^{9,10}$, Ugo Della Croce, $\mathrm{PhD}^{9,10}$, Jeffrey M Hausdorff, $\mathrm{PhD}^{2,11,12}$, Alice Nieuwboer, $\mathrm{PhD}^{1}$

${ }^{1}$ Department of Rehabilitation Sciences, KU Leuven, Tervuursevest 101, 3001 Leuven, Belgium

${ }^{2}$ Center for the Study of Movement, Cognition and Mobility, Neurological Institute, Tel Aviv Sourasky Medical Center, Tel Aviv, Israel

${ }^{3}$ Department of Neurology, Sackler School of Medicine, Tel Aviv University, Israel ${ }^{4}$ Institute of Neuroscience/Newcastle University Institute for Ageing, Clinical Ageing Research Unit, Campus for Ageing and Vitality, Newcastle University, Newcastle upon Tyne, UK

${ }^{5}$ Radboud university medical center, Donders Institute for Brain, Cognition and Behaviour, Department of Neurology, Parkinson's disease Center Nijmegen (ParC), Nijmegen, The Netherlands

${ }^{6}$ Department of Neuroscience, Rehabilitation, Ophthalmology, Genetics, Maternal, and Child Health, University of Genova, Largo Paolo Daneo 3, Genova, Italy

${ }^{7}$ IRCCS Ospedale Policlinico San Martino, Genova, Italy

${ }^{8}$ Department of Experimental Medicine, section of Human Physiology, University of Genova, Viale Benedetto XV/3, Genova, Italy

${ }^{9}$ Department of Biomedical Sciences, Bioengineering unit, University of Sassari, Sassari (SS), Italy

${ }^{10}$ Interuniversity Centre of Bioengineering of the Human Neuromusculoskeletal System, Sassari (SS), Italy

${ }^{11}$ Sagol School of Neuroscience and Sackler School of Medicine, Tel Aviv University, Tel Aviv, Israel

${ }^{12}$ Rush Alzheimer's Disease Center and Department of Orthopaedic Surgery, Rush University Medical Center, Tel Aviv, Israel 


\section{Corresponding author}

Alice Nieuwboer

Neuromotor Research Group, Department of Rehabilitation Sciences, KU Leuven

Tervuursevest 101 box 1501

3001 Leuven

Belgium

Tel: +32 16329122

Alice.nieuwboer@kuleuven.be

Word count: $3474 / 4000$

Number of figures/tables: 4/6

Supplementary: 2/4

References: 39/60 


\section{ABSTRACT}

Background: People with Parkinson's disease and freezing of gait (FOG+) have more falls, postural instability and cognitive impairment compared to FOG-.

Objective: To conduct a secondary analysis of the V-TIME study, a randomized, controlled investigation showing a greater reduction of falls after virtual reality treadmill training (TT+VR) compared to usual treadmill walking (TT) in a mixed population of fallers. We addressed whether these treadmill interventions led to similar gains in FOG+ as in FOG-.

Methods: 77 FOG+ and 44 FOG- were assigned randomly to TT+VR or TT. Participants were assessed pre- and post-training and at 6 months follow-up. Main outcome was postural stability assessed by the Mini-Best test. Falls were documented using diaries. Other outcomes included the New Freezing of Gait Questionnaire (NFOG-Q) and the Trail Making Test (TMT-B).

Results: Mini-Best scores and the TMT-B improved in both groups after training $(p=0.001)$, irrespective of study arm and FOG sub-group. However, gains were not retained at 6-months. Both FOG+ and FOG- had a greater reduction of falls after TT+VR compared to TT ( $\mathrm{p}=0.008)$. NFOG-Q scores did not change after both training modes in the FOG+ group.

Conclusions: Treadmill walking (with or without VR) improved postural instability in both FOG+ and FOG-, while controlling for disease severity differences. As found previously, TT+VR reduced falls more than TT alone, even among those with FOG. Interestingly, FOG itself was not helped by training, suggesting that although postural instability, falls and FOG are related, they may be controlled by different mechanisms. 
Falls and freezing of gait

\section{Keywords}

Parkinson's disease, freezing of gait, rehabilitation, virtual reality, falls, postural control 


\section{INTRODUCTION}

People with Parkinson's disease and freezing of gait (FOG+) are often referred by neurologists to engage in rehabilitation programs, due to the increased fall risk associated with $\mathrm{FOG}^{1,2}$. Yet, work to date does not allow drawing firm conclusions regarding training benefits in this patient cohort. As for effects on freezing severity, a recent meta-analysis suggested that non-specific exercise was not effective to alleviate freezing, as assessed with self-reported questionnaires $^{3}$. An exercise program targeting a number of fall risk factors, i.e. balance, leg strength and FOG, also did not reduce fall risk or FOG, but improved balance, mobility scores, and fear of falling ${ }^{4}$. Results of more recent work, investigating the effects of resistance versus balance training in FOG+, demonstrated no effects on balance outcomes nor on FOG severity, irrespective of training $\operatorname{arm}^{5}$. In contrast, an uncontrolled study showed that community-based group exercise for FOG+ had positive effects on balance, gait and $\mathrm{FOG}^{6}$. When gait was the specific target for exercise, it was shown that FOG+ generally responded equally well to rehabilitation compared to FOG-, when testing interventions such as dual task gait training and cued gait training ${ }^{7,8}$. However, other studies suggested that unlike FOG-, FOG+ had compromised retention of motor learning, as applied to writing training and motor sequence learning $^{9,10}$. Repeated exposure to postural perturbations during one training session on a movable balance platform also showed overall less improvements in FOG+ than FOG- ${ }^{11}$.

The manifest cognitive decline and disease progression, which partly underlie FOG, may not only explain a higher fall risk, but may also contribute to an inability to have sustained benefits from training ${ }^{12}$. This raises the question as to whether tackling both motor and cognitive functions rather than motor alone would be more beneficial for people with FOG versus those without ${ }^{7,12,13}$. 
The V-TIME study, a large EU-funded international randomized controlled trial (RCT) on fall prevention, showed a greater beneficial effect of treadmill training augmented by virtual reality $(\mathrm{TT}+\mathrm{VR})$ on fall risk compared to standard treadmill walking (TT) in fall prone older people including subjects with $\mathrm{PD}^{14}$. The V-time study was based on the very premise that combining motor-cognitive training would be better than just physical exercise to strengthen cognitive brain networks ${ }^{15}$ or the very interplay between motor and cognitive circuitry ${ }^{13}$. The VR-based intervention was also custom-made for older individuals and addressed a variety of cognitive challenges, such as executive and navigation tasks.

The above considerations motivated us to conduct a secondary analysis of the V-TIME study, investigating whether training motor-cognitive domains together (TT+VR) is more beneficial to providing treadmill training only (TT) in FOG+ as opposed to FOG-. The main outcomes of interest were balance and falls. We chose the Mini-Best test as our primary outcome of this exploratory analysis. In previous work, we found that this multifaceted test of postural control discriminated between FOG+ and FOG- ${ }^{16}$. We hypothesized that TT+VR, due to targeting motor and cognitive correlates of FOG synergistically, would lead to better or equal results in FOG+ on the Mini-Best test and falls compared to FOG-. Given earlier findings on the lack of retention in FOG+, our second research question examined whether training gains were sustained over a period of 6 months in a similar way in both subgroups. Here, we expected that FOG+ would show reduced retention compared to FOG-. We also assumed that TT+VR would have a better effect on freezing severity compared to TT in the FOG+ subgroup alone. Indirectly, therefore, this study tested that if FOG, postural instability, and falls responded comparably to training (irrespective of training mode) that this would point to similar underlying neural substrates among these outcomes. 


\section{METHODS}

\section{Study design}

This analysis was based on a subset of subjects who participated in the randomized controlled trial entitled "Virtual reality Treadmill combined Intervention to improve Mobility and reduce falls in the Elderly" (FP7 project V-TIME-278169). Five clinical partners were involved from Israel (Tel Aviv Sourasky Medical Center), Belgium (Katholieke Universiteit Leuven), United Kingdom (Newcastle University), Italy (University of Genoa) and The Netherlands (Radboud University Medical Center Nijmegen). Eligible subjects were assigned randomly to an intervention group consisting of treadmill training plus VR (TT+VR) or a control group entailing treadmill training alone VR (TT). Originally, the dataset consisted of 130 PD patients. The current sub-analysis involved 121 PD patients, as FOG-scores from 9 patients were unavailable. Patients were assessed 1 week before training, 1 week after the 6week intervention, and 6 months after training.

\section{Participants}

77 Patients with FOG (FOG+) and 44 without FOG (FOG-) were included in this analysis. The FOG+-group was defined based a score $>1$ on the New Freezing of Gait Questionnaire $^{17}$. Recruitment took place between January 2012 and January 2015. All participants provided written informed consent in accordance to the Declaration of Helsinki. The study was approved by ethics committees in each of the five clinical sites separately and registered under trial NCT01732653.

Participants were included if they were between 60 and 90 years of age, had been diagnosed based on the UK Brain Bank criteria, Hoehn and Yahr (H\&Y) stage II or III, were 
on anti-Parkinsonian medication, were able to walk at least five minutes without assistance, had adequate hearing and vision (as evaluated by the whisper test and Snellen test respectively) and had experienced two or more falls in the previous six months (self-reported). Exclusion criteria comprised psychiatric co-morbidity, clinical diagnosis of dementia or other severe cognitive impairment (Mini-Mental State Examination (MMSE) score <24), history of stroke, traumatic brain injury or neurological disorders other than PD, rheumatic and orthopedic diseases, acute lower back or lower extremity pain, peripheral neuropathy and an inability to comply with the training.

\section{Training}

A detailed description of the intervention and the RCT findings were published previously ${ }^{14,18}$. In brief, participants exercised three times per week over a period of six weeks with each session lasting approximately 45 minutes in both training arms. Exercise programs were supervised by therapists (mean years of experience 5.2 \pm 3.2 years, range: 1-9 years), who were trained in the delivery methods prior to the first patient-in. Regular fidelity checks were performed across centers to ensure uniformity ${ }^{18}$. Both arms received walking exercises on a treadmill, whereby gait speed and walking duration were progressively increased throughout the six-week intervention using pre-determined levels and criteria for progression ${ }^{18}$. In the beginning of each training week, over-ground gait speed was measured over 10 meters. Based on this, treadmill speed was set at $80 \%$ of over-ground gait velocity in the first week. This was increased to $90 \%$ in the second week and was aimed to go up with $10 \%$ from the third week. Walking duration was also increased from 20 to 45 minutes throughout the six weeks and a gradual reduction of handrail support was introduced until none was needed. As for the VR progression milestones, every week obstacle levels were increased in height and depth, visibility was reduced from daylight to darkness, distractors in the environment were increased 
from calm to busy and navigation signposts were reduced from many to none to stimulate memory functions. Progression was, however, subject to participant's performance and ability $^{18}$.

The VR intervention was designed to tackle fall risk by training obstacle negotiation in a complex and interactive virtual environment. It included obstacles appearing at different sizes, frequencies and visibility, requiring step adjustments in both height and length. In addition, the VR environment posed a cognitive load using a navigation task and by adding visual and auditory distractors, stimulating attention, executive function, dual-tasking, planning and response selection. Visual and auditory feedback on performance and results were provided during training and summarized at the end of each session in both arms of the study. Details on the amount of visual and auditory feedback as derived from the VR intervention were described in previous papers ${ }^{14,18}$. Training progression was modulated by gradually increasing the walking speed, duration and difficulty levels in the VR environment.

\section{Outcomes}

Testing was conducted by blinded testers for group allocation, using standardized guidelines across centers. Training occurred in a separate room from testing to ensure that blinding was maintained. The trainer also delivered the intervention on an individual basis and at separate moments to avoid contamination between groups.

All assessments were performed while when patients were in the on-medication state. Following the first medication anamnesis, test times were determined to allow testing in a stable on-period, which was standardized for subsequent tests. Testers also telephoned participants a day prior to each test to verify time of medication intake. For this analysis, we focused on the Mini Balance Evaluation System Test (Mini-BEST), as pathology in static and dynamic postural control likely underlies both FOG and fall risk. The validity and reliability for this 
scale in PD are well-established ${ }^{19,20}$. Other freezing-related outcomes were number of falls, assessed pre-training (by self-report of retrospective fall frequency) and at 6 months follow-up. During the 6 months after training, falls were recorded through a falls calendar, which was provided as a paper version, web-based calendar or a smartphone application according to individual preference. Each fall was directly indicated on the calendar. FOG severity was assessed with the New Freezing of Gait Questionnaire (NFOG-Q) ${ }^{17}$.

Secondary outcomes included executive function as assessed by the Trail Making Test part B (TMT-B). We chose this test for its psychometric properties ${ }^{21}$ and its ability to discriminate between FOG+ and $\mathrm{FOG}_{-}{ }^{22}$. Other secondary outcomes included two tests of overall mobility (Short Physical Performance Battery, SPPB; Four Square Step Test, FSST), balance confidence (Falls Efficacy Scale International, FES-I) and daily activity (Physical Activity for the Elderly, PASE). Descriptor variables included age, gender, years of education, global cognitive function using the MMSE and Montreal Cognitive Assessment (MoCA) and disease severity, assessed by Hoehn \& Yahr (H\&Y) staging, Unified Disease Parkinson's Disease Rating Scale motor part (III) (MDS-UPDRS-III) and disease duration.

\section{Statistical analyses}

Statistical analysis was performed using IBM SPSS software (version 22). Demographic characteristics were compared between groups using Independent t-tests and Chi-square statistics. Generalized linear mixed-effects models were used with Group (FOG+/FOG-), Training arm (TT/TT+VR) and Time (pre, post, 6 months follow-up) as fixed effects. We also used UPDRS-III as a continuous covariate to adjust for differences in disease severity, whilst avoiding co-linearity. As this analysis was a secondary analysis, no a-priori power analysis was carried out. All models controlled for within-subject differences by including participants as 
random effects. Falls were examined using a negative binomial regression, with no exposure variable included. For all analyses, alpha was set at 0.05 and post-hoc analyses were carried out using Bonferroni tests. 


\section{RESULTS}

Group demographics are presented in Table 1. Age, cognition, gender and fall rates were similar in FOG+ and FOG- at baseline. The FOG+ group had worse disease profiles, as might be expected, shown by higher disease duration $(\mathrm{p}=0.008), \mathrm{H} \& \mathrm{Y}(\mathrm{p}=<0.001)$ and UPDRS-III scores $(\mathrm{p}=0.02)$. Furthermore, FOG+ had lower balance scores on the Mini-BEST $(p=0.044)$ and more fear of falling $(\mathrm{p}=0.006)$.

The distribution of the two FOG groups differed between both training groups. Of the FOG+ group, 43 were in TT versus 34 in TT+VR. Of the FOG- group, 16 were included in TT and 28 in TT+VR. No differences were present between TT and TT+VR training arms at baseline. Within each FOG-subgroup, participants in the two training arms were well-matched with respect to all baseline characteristics (Table 1/supp). Only within FOG-, disease duration was slightly longer in the VR compared to those in the TT arm $(\mathrm{p}=0.044)$.

\section{Effect on balance, falls and FOG}

An overview of these results is provided in Table 2 and presented in Figures 1 and 2. Overall, no three-way interactions between, time, training modes and FOG-subgroups were found and most results indicated significant time effects only after controlling for disease severity. The primary outcome, balance performance as evaluate by the Mini-BEST scores, improved in both groups immediately after training $(\mathrm{p}=0.001)$, irrespective of training arm and subgroup. However, improvements were not retained at 6 months and declined in a similar fashion across subgroups and training arms.

A training Arm*Time interaction $(\mathrm{p}=0.027)$ did show a significantly greater reduction of falls in the TT+VR group in both FOG+ and FOG- as compared to the TT group ( $\mathrm{p}=0.008$ ). Fall rates were significantly lower after 6 months than at baseline in the VR group (5.39 [95\% 
CI 4.09-7.21]; $\mathrm{p}<0.001)$, while this was not significantly different in the TT group (11.95 [95\%

CI 8.76-16.31]; $\mathrm{p}=0.099$ ). Another main effect for fall rate was found for the FOG-subgroup $(\mathrm{p}<0.001)$, indicating that FOG+ had overall higher fall rates even after correcting for disease severity (slightly different from the baseline unadjusted fall rates in Table 1). When we analyzed the effects of training on FOG severity in FOG+ only, the NFOG-Q scores did not improve after both training modes. Additionally, FOG severity increased at 6 months compared to post-training assessment $(\mathrm{p}=0.037)$ and got worse beyond baseline levels.

\section{Effects on other outcomes}

The results of training on the secondary outcomes are presented in Table 2. As above, no three-way interactions between, time, training modes and FOG-subgroups were apparent. A significant effect of time was found for executive function (TMT-B scores) $(\mathrm{p}<0.001)$, indicating that both groups benefited equally from both training modes. Overall mobility performance (SPPB-scores) significantly improved immediately after training in both arms $(\mathrm{p}=0.001)$, but improvements were not retained at 6 months $(\mathrm{p}=0.030)$. Furthermore, SPPB scores were generally higher in the TT+VR group compared to the TT group ( $\mathrm{p}=0.026)$, but no interactions were found with time or subgroup. Fear of falling, as assessed with the FES-I, was overall higher in FOG+ $(\mathrm{p}=0.004)$, but this was not influenced by training $(\mathrm{p}=0.388)$, nor by training mode $(\mathrm{p}=0.178)$. No intervention-related changes were found on other mobility measures, such as the FSST and PASE.

\section{DISCUSSION}

This study investigated the effectiveness of treadmill training with (TT+VR) and without virtual reality (TT) on balance and falls differentiating between PD patients with and without FOG. We hypothesized that TT+VR would be more, or at least as, beneficial for FOG+ 
compared to FOG-. In contrast, we found that FOG+ gained as much as FOG- from TT and $\mathrm{TT}+\mathrm{VR}$, as their postural instability, executive function and general mobility improved to an equal degree. We also found that despite more severe clinical profiles, higher fall rates and more fear of falling than FOG-, FOG+ also reported a greater reduction of fall rates after TT+VR compared to TT. The second hypothesis, that FOG+ would show less retention of training effects compared to FOG-, was also rejected as both groups consistently showed loss of training benefits after 6 months. As for the effects on FOG itself, which could only be determined in the FOG+ group, neither TT+VR nor TT influenced this symptom. Disappointingly, freezing severity even increased considerably in both training arms at the sixmonth follow-up.

In line with what was presented for a wider group of fall-prone older adults ${ }^{14}$ both FOGand FOG+ improved postural instability and cognitive function after both training modes, but did not retain these effects after cessation of the intervention. In FOG+, executive dysfunction is usually more pronounced compared to FOG- $^{12}$. Yet, here we could establish that complex training addressing motor-cognitive integration was as beneficial for this subgroup, at least in the short term. This may partly be explained by the fact that at baseline FOG+ and FOG- had similar cognitive scores on generic tests (MoCA and MMSE). The results also show, overall, that long-term retention of treadmill intervention was equally impaired in both PD subgroups. Other studies indicated shorter retention of motor learning in FOG+ compared to FOG- after complex dual-task interventions and writing training ${ }^{9}$. Providing motor-cognitive rehabilitation on a regular basis and for a longer period might improve long-term retention. However, the optimal frequency and intensity to sustain training gains is not yet clear in PD and would need more frequent test moments. Recent study showed that, irrespective of the presence of FOG, 
the effects of intensive balance training for ten weeks were predicted to disappear completely after 14 months without training ${ }^{23}$.

The rate of falls was significantly lower at 6 months follow-up, with a larger effect of TT+VR over TT alone. Training with VR may have led to increased attention while negotiating obstacles in the real world, as a result of which tripping risks may have been reduced, the most common cause of near-falls among fallers with $\mathrm{PD}^{24}$. The VR environment also stimulated cognitive and visual processing, such as focused attention, response selection and planning while walking ${ }^{18}$. As walking is a complex task, requiring integration of motor, cognitive, perceptual resources for dynamic postural control and bilateral coordination ${ }^{25}$, combined motor-cognitive training may have enhanced these processes in both FOG+ and FOG-. Recent frameworks for rehabilitation, specifically targeted at FOG, promote integration of cognitive and motor components ${ }^{6,26,27,28}$. The present results strengthen the use of such approaches in clinical practice, at least for ameliorating falling, even when targeting extremely fall-prone individuals such as those with FOG.

Although falls, postural control and FOG co-occur and are likely etiologically intertwined $^{29}$, the self-reported measure of FOG, the NFOG-Q, did not improve after training, unlike postural instability (the Mini-Best test) and falls. Most strikingly, FOG deteriorated significantly with time beyond baseline levels, which was not the case for falls and postural instability as tested in both FOG+ and FOG-. Although speculative, these discrepant results support the notion that these phenomena are governed by partially overlapping, but also distinct neural pathways ${ }^{30,31}$. We recently published a review of the literature to determine whether there is evidence to claim that FOG and postural instability have common mechanisms ${ }^{29}$. We found that the findings on medication suggested that non-dopaminergic mechanisms were even 
more involved in postural instability than in FOG. Common neural pathway for modulation of FOG and postural instability likely incorporate the mesencephalic locomotor (MLR) region and the cerebellum ${ }^{32,33}$. Still, motor imagery data revealed that the latero-rostral part of the MLR might regulate balance, whereas the medial-caudal MLR appeared more involved in gait ${ }^{34}$. The effectors of balance and gait, primarily concerning the trunk and leg muscles have different somatotopic representations in various brain regions, which would also account for the limited overlap between the two phenomena. All this, and the fact that training had such contradictory effects would suggest that the occurrence of FOG-episodes are etiologically different than falls, although postural instability and cognitive capacity mediate both these phenomena.

Our results are different than those of a series of studies that tested the effects of interventions specifically designed to alleviate FOG. A recent pilot study on the efficacy of a 6-week supervised training to reduce FOG demonstrated consistent improvements of FOG, gait, and balance ${ }^{6}$. Similarly, 12 sessions of curved treadmill walking led to gains in both FOG and functional walking maintained for at least one month ${ }^{35}$. Also, 8 sessions of 20 -minutes dual motor-cognitive virtual reality training by stepping-in-place led to a reduction in the number of FOG episodes ${ }^{36}$. Various modes of action observation, by watching FOG-alleviating videos as well as undergoing physical training, led to significant improvements in FOG severity with good retention at follow-up ${ }^{37-39}$. Together, these results could signify that motor-cognitive therapy needs to be specifically designed to alleviate FOG. In contrast, the TT+VR training of the present study, although relevant for FOG, was designed to target falls and mobility, but was not specifically aimed at tackling FOG. The evidence above highlights the importance of tailored and intensive programs, addressing both FOG triggers and modifiers, in order to alleviate FOG. 


\section{Limitations}

Falls were reduced when assessed over a total period of six months. However, measures of postural instability were captured immediately after training as well as at follow-up. Future work should incorporate more frequent and parallel measures of fall rates, balance, and FOG scores to understand how the changes over time in these outcomes are interrelated. In addition, it is possible that changes in FOG occurred which were not captured by the NFOG-Q, as responsiveness of this scale is still unknown. Although the groups were relatively large, this secondary analysis was not powered for this sub-analysis and did not account for center effects, as none were found in the previous analysis of the V-time study ${ }^{14}$. Therefore, our findings could have suffered from type II error. In addition, the unequal group sizes between FOG+ and FOG, especially within training arms, must also be acknowledged.

\section{Conclusions}

Despite the higher risk of falls, people with FOG benefited equally from treadmill training with and without VR with regards to improving their balance and reducing fall risk compared to their non-freezing counterparts. Overall, the present exploratory findings identified a similar training potential in FOG+ and FOG-, as well as a similar decline of practice effects after a period without training. Interestingly, freezing itself was not alleviated by combined motor and cognitive training and deteriorated importantly over the 6 months followup period. While the current positive results of treadmill training in people with FOG are encouraging, they also point to the need to specifically target rehabilitation to the origins of FOG in order to be able to modify this complex problem.

\section{Declaration of Interest}


$\mathrm{JMH}$ has received grant support from the NIH, the Michael J Fox Foundation for Parkinson's Research, the EU (H2020), the United States Israel Binational Science Foundation, the Israeli Science Foundation and the National Multiple Sclerosis Society. JM and AM submitted a patent application on the use of virtual reality, the intellectual property rights for which are held by the Tel Aviv Medical Center. JMH has or currently serves on the Movement Disorders Society Technology Task Force and on Michael J Fox Foundation task force on gait, on the board of the International Society for the Measurement of Physical Behavior, and on advisory boards for Sanofi and Biogen.

AM has received grant support from the Michael J Fox Foundation for Parkinson's Research, the Israeli Science Foundation and Ministry of Science and Technology. She serves on the Gait Advisory committee for Michael J Fox Foundation for Parkinson's Research and, on advisory boards for Biogen.

BRB currently serves as Associate Editor for the Journal of Parkinson's disease, serves on the editorial of Practical Neurology and Digital Biomarkers, has received honoraria from serving on the scientific advisory board for Abbvie, Biogen, UCB and Walk with Path, has received fees for speaking at conferences from AbbVie, Zambon, Roche, GE Healthcare and Bial, and has received research support from the Netherlands Organization for Scientific Research, the Michael J Fox Foundation, UCB, Abbvie, the Stichting Parkinson Fonds, the Hersenstichting Nederland, the Parkinson's Foundation, Verily Life Sciences, Horizon 2020, the Topsector Life Sciences and Health, and the Parkinson Vereniging. 


\section{Acknowledgements}

This research was funded by the European Commission (FP7 project V-TIME278169). We cordially thank all the V-TIME participants for engaging in the study. 


\section{References}

1. Fasano A, Canning CG, Hausdorff JM, Lord S, Rochester L. Falls in Parkinson's disease: A complex and evolving picture. Mov Disord. 2017;32(11):1524-1536. doi:10.1002/mds.27195.

2. Bloem BR, Hausdorff JM, Visser JE, Giladi N. Falls and freezing of gait in Parkinson's disease: a review of two interconnected, episodic phenomena. Mov Disord. 2004;19(8):871-884. doi:10.1002/mds.20115.

3. Ni M, Hazzard JB, Signorile JF, Luca C. Exercise Guidelines for Gait Function in Parkinson's Disease: A Systematic Review and Meta-analysis. Neurorehabil Neural Repair. 2018;32(10):872-886. doi:10.1177/1545968318801558.

4. Canning CG, Sherrington C, Lord SR, et al. Exercise for falls prevention in Parkinson disease: A randomized controlled trial. Neurology. 2015;84(3):304-312. doi:10.1212/WNL.0000000000001155.

5. Schlenstedt C, Paschen S, Seuthe J, et al. Moderate Frequency Resistance and Balance Training Do Not Improve Freezing of Gait in Parkinson's Disease: A Pilot Study. Front Neurol. 2018;9. doi:10.3389/fneur.2018.01084.

6. Rawson KS, Creel P, Templin L, Horin AP, Duncan RP, Earhart GM. Freezing of Gait Boot Camp: feasibility, safety and preliminary efficacy of a community-based group intervention. Neurodegener Dis Manag. 2018;8(5):307-314. doi:10.2217/nmt-2018-0022.

7. Strouwen C, Molenaar EALM, Münks L, et al. Training dual tasks together or apart in Parkinson's disease: Results from the DUALITY trial. Mov Disord. 2017;32(8):1201-1210. doi:10.1002/mds.27014.

8. Nieuwboer A, Kwakkel G, Rochester L, et al. Cueing training in the home improves gait-related mobility in Parkinson's disease: the RESCUE trial. J Neurol Neurosurg Psychiatry. 2007;78(2):134-140. doi:10.1136/jnnp.200X.097923.

9. Heremans E, Nackaerts E, Vervoort G, Broeder S, Swinnen SP, Nieuwboer A. Impaired retention of motor learning of writing skills in patients with Parkinson's disease with freezing of gait. PLoS One. 2016;11(2). doi:10.1371/journal.pone.0148933.

10. Vandenbossche J, Deroost N, Soetens E, et al. Impaired implicit sequence learning in Parkinson's disease patients with freezing of gait. Neuropsychology. 2013;27(1):28-36. doi:10.1037/a0031278. 
11. Peterson DS, Horak FB. Effects of freezing of gait on postural motor learning in people with Parkinson's disease. Neuroscience. 2016;334:283-289. doi:10.1016/j.neuroscience.2016.08.017.

12. Heremans E, Nieuwboer A, Spildooren J, et al. Cognitive aspects of freezing of gait in Parkinson's disease: a challenge for rehabilitation. J Neural Transm. 2013;120(4):543-557. doi:10.1007/s00702-012-0964-y.

13. Li KZH, Bherer L, Mirelman A, Maidan I, Hausdorff JM. Cognitive Involvement in Balance, Gait and Dual-Tasking in Aging: A Focused Review From a Neuroscience of Aging Perspective. Front Neurol. 2018;9:913. doi:10.3389/fneur.2018.00913.

14. Mirelman A, Rochester L, Maidan I, et al. Addition of a non-immersive virtual reality component to treadmill training to reduce fall risk in older adults (V-TIME): a randomised controlled trial. Lancet. 2016. doi:10.1016/S0140-6736(16)31325-3.

15. Maidan I, Rosenberg-Katz K, Jacob Y, Giladi N, Hausdorff JM, Mirelman A. Disparate effects of training on brain activation in Parkinson disease. Neurology. 2017;89(17):1804-1810. doi: 10.1212/WNL.0000000000004576.

16. Bekkers EMJ, Dijkstra BW, Dockx K, Heremans E, Verschueren SMP, Nieuwboer A. Clinical balance scales indicate worse postural control in people with Parkinson's disease who exhibit freezing of gait compared to those who do not: A meta-analysis. Gait Posture. 2017;56:134-140. doi:10.1016/j.gaitpost.2017.05.009.

17. Nieuwboer A, Rochester L, Herman T, et al. Reliability of the new freezing of gait questionnaire: agreement between patients with Parkinson's disease and their carers. Gait Posture. 2009;30(4):459-63. doi:10.1016.j.gaitpost.2009.07.108.

18. Mirelman A, Rochester L, Reelick M, et al. V-TIME: a treadmill training program augmented by virtual reality to decrease fall risk in older adults: study design of a randomized controlled trial. BMC Neurol. 2013;13:15. doi:10.1186/1471-2377-1315.

19. Löfgren N, Benka Wallén M, Sorjonen K, Conradsson D, Franzén E. Investigating the Mini-BESTest's construct validity in elderly with Parkinson's disease. Acta Neurol Scand. 2017;135(6):614-621. doi:10.1111/ane.12640.

20. Löfgren N, Lenholm E, Conradsson D, Stahle A, Franzen E. The Mini-BESTest - a clinically reproducible tool for balance evaluations in mild to moderate Parkinson's disease? BMC Neurol. 2014;14:235. doi:10.1186/s12883-014-0235-7. 
21. Sánchez-Cubillo I, Periáñez JA, Adrover-Roig D, et al. Construct validity of the Trail Making Test: Role of task-switching, working memory, inhibition/interference control, and visuomotor abilities. J Int Neuropsychol Soc. 2009;15(3):438-450. doi:10.1017/S1355617709090626.

22. Martens KAE, Hall JM, Gilat M, Georgiades MJ, Walton CC, Lewis SJG. Anxiety is associated with freezing of gait and attentional set-shifting in Parkinson's disease: A new perspective for early intervention. Gait Posture. 2016;49:431-436. doi:10.1016/j.gaitpost.2016.07.182.

23. Wallén MB, Hagströmer M, Conradsson D, Sorjonen, Franzén E. Long-term effects of highly challenging balance training in Parkinson's disease-a randomized controlled trial. Clin Rehabil. 2018;32(11):1520-1529. doi:10.1177/0269215518784338.

24. Gazibara T, Kisic Tepavcevic D, Svetel M, et al. Near-falls in people with Parkinson's disease: Circumstances, contributing factors and association with falling. Clin Neurol Neurosurg. 2017;161:51-55. doi:10.1016/j.clineuro.2017.08.008.

25. Amboni M, Barone P, Hausdorff JM. Cognitive contributions to gait and falls: Evidence and implications. Mov Disord. 2013;28(11):1520-1533. doi:10.1002/mds.25674.

26. Peterson DS, King LA, Cohen RG, Horak FB. Cognitive Contributions to Freezing of Gait in Parkinson Disease: Implications for Physical Rehabilitation. Phys Ther. 2015;96(5):659-670. doi:10.2522/ptj.20140603.

27. King LA, Peterson DS, Mancini M, et al. Do cognitive measures and brain circuitry predict outcomes of exercise in Parkinson Disease: A randomized clinical trial. BMC Neurol. 2015;15(1). doi:10.1186/s12883-015-0474-2.

28. Dagan M, Herman T, Harrison R et al. Multitarget transcranial direct current stimulation for freezing of gait in Parkinson's disease. Mov Disord. 2018; 33(4):642646. doi:10.1002/mds.27300.

29. Bekkers EMJ, Dijkstra BW, Heremans E, Verschueren SMP, Bloem BR, Nieuwboer A. Balancing between the two: Are freezing of gait and postural instability in Parkinson's disease connected? Neurosci Biobehav Rev. 2018;94:113125. doi:10.1016/j.neubiorev.2018.08.008. 
30. Nonnekes J, de Kam D, Oude Nijhuis LB, et al. StartReact effects support different pathophysiological mechanisms underlying freezing of gait and postural instability in Parkinson's disease. PLoS One. 2015;10(3):e0122064. doi:10.1371/journal.pone.0122064.

31. Factor SA, Steenland NK, Higgins DS, et al. Postural instability/gait disturbance in Parkinson's disease has distinct subtypes: an exploratory analysis. J Neurol Neurosurg Psychiatry. 2011;82:564-568. doi:10.1136/jnnp.2010.222042.

32. Gilat M, Dijkstra BW, D'Cruz N, Nieuwboer A, Lewis SJG. Functional MRI to Study Gait Impairment in Parkinson's Disease: a Systematic Review and Exploratory ALE Meta-Analysis. Curr Neurol Neurosci Rep. 2019;19(8):49. doi: $10.1007 / \mathrm{s} 11910-019-0967-2$

33. Lewis SJ, Shine JM. The Next Step:

A Common Neural Mechanism for Freezing of Gait.

Neuroscientist. 2016;22(1):72-82. doi: 10.1177/1073858414559101.

34. Ferraye MU, Debû B, Heil L, Carpenter M, Bloem BR, Toni I. Using motor imagery to study the neural substrates of dynamic balance. PLoS One. 2014;9(3):e91183. doi: 10.1371/journal.pone.0091183

35. Cheng FY, Yang YR, Wu YR, Cheng SJ, Wang RY. Effects of curved-walking training on curved-walking performance and freezing of gait in individuals with Parkinson's disease: A randomized controlled trial. Park Relat Disord. 2017;43:2026. doi:10.1016/j.parkreldis.2017.06.021.

36. Killane I, Fearon C, Newman L, et al. Dual motor-cognitive virtual reality training impacts dual-task performance in freezing of gait. IEEE J Biomed Heal Informatics. 2015;19(6):1855-1861. doi:10.1109/JBHI.2015.2479625.

37. Mezzarobba S, Grassi M, Pellegrini L, et al. Action observation plus sonification. A novel therapeutic protocol for Parkinson's patient with freezing of gait. Front Neurol. 2018;8(JAN). doi:10.3389/fneur.2017.00723.

38. Pelosin E, Avanzino L, Bove M, Stramesi P, Nieuwboer A, Abbruzzese G. Action observation improves freezing of gait in patients with Parkinson's disease. Neurorehabil Neural Repair. 2010;24(8):746-752. doi:10.1177/1545968310368685. 
39. Pelosin E, Barella R, Bet C, et al. Effect of Group-Based Rehabilitation Combining Action Observation with Physiotherapy on Freezing of Gait in Parkinson's Disease. Neural Plast. 2018;2018:1-7. doi:10.1155/2018/4897276. 
Table 1. Demographic characteristics of PD-groups and training arms

\begin{tabular}{lccr|ccc}
\hline & FOG+ & FOG- & $\boldsymbol{p}$-value & $\begin{array}{c}\text { TT } \\
\mathbf{n}=\mathbf{5 9}\end{array}$ & $\begin{array}{c}\text { TT+VR } \\
\mathbf{n}=\mathbf{6 2}\end{array}$ & $\boldsymbol{p}$-value \\
\hline Age & $70.57 \pm 6.04$ & $71.66 \pm 6.3$ & 0.351 & $70.86 \pm 6.0$ & $71.06 \pm 6.3$ & 0.859 \\
Education & $13.22 \pm 4.3$ & $13.41 \pm 4.8$ & 0.823 & $13.26 \pm 4.4$ & $13.32 \pm 4.3$ & 0.936 \\
Gender (m/f) & $49 / 28$ & $25 / 19$ & 0.459 & $37 / 22$ & $37 / 25$ & 0.732 \\
Disease duration (yrs) & $10.43 \pm 6.7$ & $7.25 \pm 5.1$ & $\mathbf{0 . 0 0 8}$ & $9.55 \pm 7.2$ & $9.05 \pm 5.5$ & 0.669 \\
H\&Y & $2.58 \pm 0.4$ & $2.23 \pm 0.4$ & $<\mathbf{0 . 0 0 1}$ & $2.49 \pm 0.5$ & $2.42 \pm 0.5$ & 0.401 \\
MMSE & $28.22 \pm 1.6$ & $27.73 \pm 1.6$ & 0.106 & $28.34 \pm 1.5$ & $27.76 \pm 1.7$ & $\mathbf{0 . 0 4 7}$ \\
NFOG-Q & $15.87 \pm 6.7$ & $0.00 \pm 0.0$ & $<\mathbf{0 . 0 0 1}$ & $11.69 \pm 9.3$ & $8.58 \pm 9.3$ & 0.069 \\
MoCA & $24.01 \pm 3.8$ & $24.14 \pm 4.4$ & 0.872 & $24.27 \pm 3.5$ & $23.85 \pm 4.5$ & 0.572 \\
UPDRS-III & $31.83 \pm 13.2$ & $26.11 \pm 12.2$ & $\mathbf{0 . 0 2}$ & $29.37 \pm 13.2$ & $30.11 \pm 13.1$ & 0.757 \\
Mini-BEST (/28) & $20.44 \pm 5.8$ & $22.68 \pm 5.9$ & $\mathbf{0 . 0 4 4}$ & $21.00 \pm 6.1$ & $21.50 \pm 5.8$ & 0.643 \\
FES-I & $36.74 \pm 11.7$ & $30.65 \pm 10.3$ & $\mathbf{0 . 0 0 6}$ & $36.39 \pm 11.4$ & $32.72 \pm 11.7$ & 0.088 \\
Falls & $26.29 \pm 65.1$ & $16.20 \pm 54.3$ & 0.387 & $26.44 \pm 69.1$ & $18.98 \pm 53.3$ & 0.506 \\
Group (TT/ TT+VR) & & & & & & \\
(FOG+/FOG-) & $43 / 34$ & $16 / 28$ & $\mathbf{0 . 0 3 9}$ & $16 / 43$ & $28 / 34$ & $\mathbf{0 . 0 3 9}$ \\
\hline
\end{tabular}

Abbreviations: FES-I: Falls Efficacy Scale-International; FOG+: patients with freezing of gait; FOG-: patients without freezing of gait; H\&Y: Hoehn \& Yahr; Mini-BEST: Mini-Balance Evaluation System Test; MMSE: Mini-Mental State Examination; MoCA: Montreal Cognitive Assessment; NFOG-Q: New Freezing of Gait Questionnaire; PD: Parkinson's Disease; TT: Treadmill training; TT+VR: Treadmill training plus virtual reality; UPDRS-III: Unified Parkinson's Disease Rating Scale motor part (III). 
Table 2. Primary and secondary outcome measures pre- and post-intervention and at 6 months followup.

\begin{tabular}{|c|c|c|c|c|c|c|c|}
\hline & & & & & & p-value & \\
\hline & & TT & $\mathrm{TT}+\mathrm{VR}$ & $\begin{array}{c}\text { Contrast estimate } \\
(95 \% \mathrm{Cl})\end{array}$ & $\begin{array}{l}\text { p-value } \\
\text { Time }\end{array}$ & $\begin{array}{l}\text { Training } \\
\text { arm }\end{array}$ & $\begin{array}{l}\text { p-value } \\
\text { FOG+/- }\end{array}$ \\
\hline Mini-BEST & & & & & 0.002 & 0.407 & 0.404 \\
\hline & Pre-training & 21.27 & 21.62 & $0.333(-1.564-2.230)$ & & & \\
\hline & Post-training & 22.26 & 23.51 & $1.258(-0.697-3.212)$ & & & \\
\hline & 6 month follow-up & 21.76 & 22.37 & $0.616(-1.586-2.817)$ & & & \\
\hline NFOG-Q ${ }^{\dagger}$ & & & & & 0.036 & 0.832 & n.a. \\
\hline & Pre-training & 16.06 & 15.44 & $-0.623(-3.876-2.630)$ & & & \\
\hline & Post-training & 14.89 & 15.75 & $0.852(-2.419-4.124)$ & & & \\
\hline & 6 month follow-up & 16.87 & 17.64 & $0.776(-2.574-4.127)$ & & & \\
\hline SPPB & & & & & 0.001 & 0.026 & 0.214 \\
\hline & Pre-training & 8.132 & 8.815 & $0.684(-0.189-1.556)$ & & & \\
\hline & Post-training & 8.776 & 9.423 & $0.648(-0.154-1.449)$ & & & \\
\hline & 6 month follow-up & 8.059 & 9.248 & $1.189(0.310-2.068)$ & & & \\
\hline FSST & & & & & 0.388 & 0.829 & 0.468 \\
\hline & Pre-training & 14.85 & 13.91 & $-0.943(-3.472-1.586)$ & & & \\
\hline & Post-training & 13.75 & 14.55 & $0.805(-2.460-4.069)$ & & & \\
\hline & 6 month follow-up & 14.04 & 13.39 & $-0.653(-3.254-1.949)$ & & & \\
\hline TMT-B & & & & & $<0.001$ & 0.870 & 0.195 \\
\hline & Pre-training & 171.05 & 173.87 & $2.822(-27.46-33.103)$ & & & \\
\hline & Post-training & 153.01 & 150.67 & $-2.335(-29.192-24.552)$ & & & \\
\hline & 6 month follow-up & 158.44 & 164.3 & $6.088(-23.146-35.322)$ & & & \\
\hline FES-I & & & & & 0.388 & 0.178 & 0.004 \\
\hline & Pre-training & 35.033 & 32.799 & $-2.234(-6.399-1.931)$ & & & \\
\hline & Post-training & 34.249 & 32.49 & $-1.459(-5.935-2.418)$ & & & \\
\hline & 6 month follow-up & 34.438 & 30.364 & $-3.674(-8.149-0.801)$ & & & \\
\hline PASE & & & & & 0.13 & 0.86 & 0.315 \\
\hline & Pre-training & $90.18^{*}$ & 93.12 & $3.031(-20.566-26.629)$ & & & \\
\hline & Post-training & 81.59 & 91.46 & $9.874(-10.994-30.743)$ & & & \\
\hline & 6 month follow-up & 100.53 & 92.87 & $-7.661(-31.657-16.334)$ & & & \\
\hline
\end{tabular}

Values represent estimated means (corrected for UPDRS-III). P-values show main effects of time, training arm and FOG-subgroup. ${ }^{\star}=$ Significant time*training interaction, $\mathrm{p}=0.027{ }^{\dagger}=$ applies to FOG-group only (uncorrected).

Abbreviations: Mini-BEST: Mini-Balance Evaluation System Test; TMT-B: Trail Making Test part B; NFOG-Q: New Freezing of Gait Questionnaire; SPPB: Short Physical Performance Battery; FSST: Four Square Step Test; FES-I: Falls Efficacy Scale-International; PASE: Physical Activity Scale for the Elderly. 
Figure 1. Results on balance, executive function, falls and freezing of gait in the treadmill training with virtual reality (TT+VR) and treadmill training (TT) arms pre- and post-training and at 6 months follow-up. No differences between FOG-subgroups were apparent and therefore not individually displayed. Estimated means and standard errors are shown. ${ }^{*} \mathrm{p}<0.05$, $* * \mathrm{p}<0.01, * * * \mathrm{p}<0.001$.
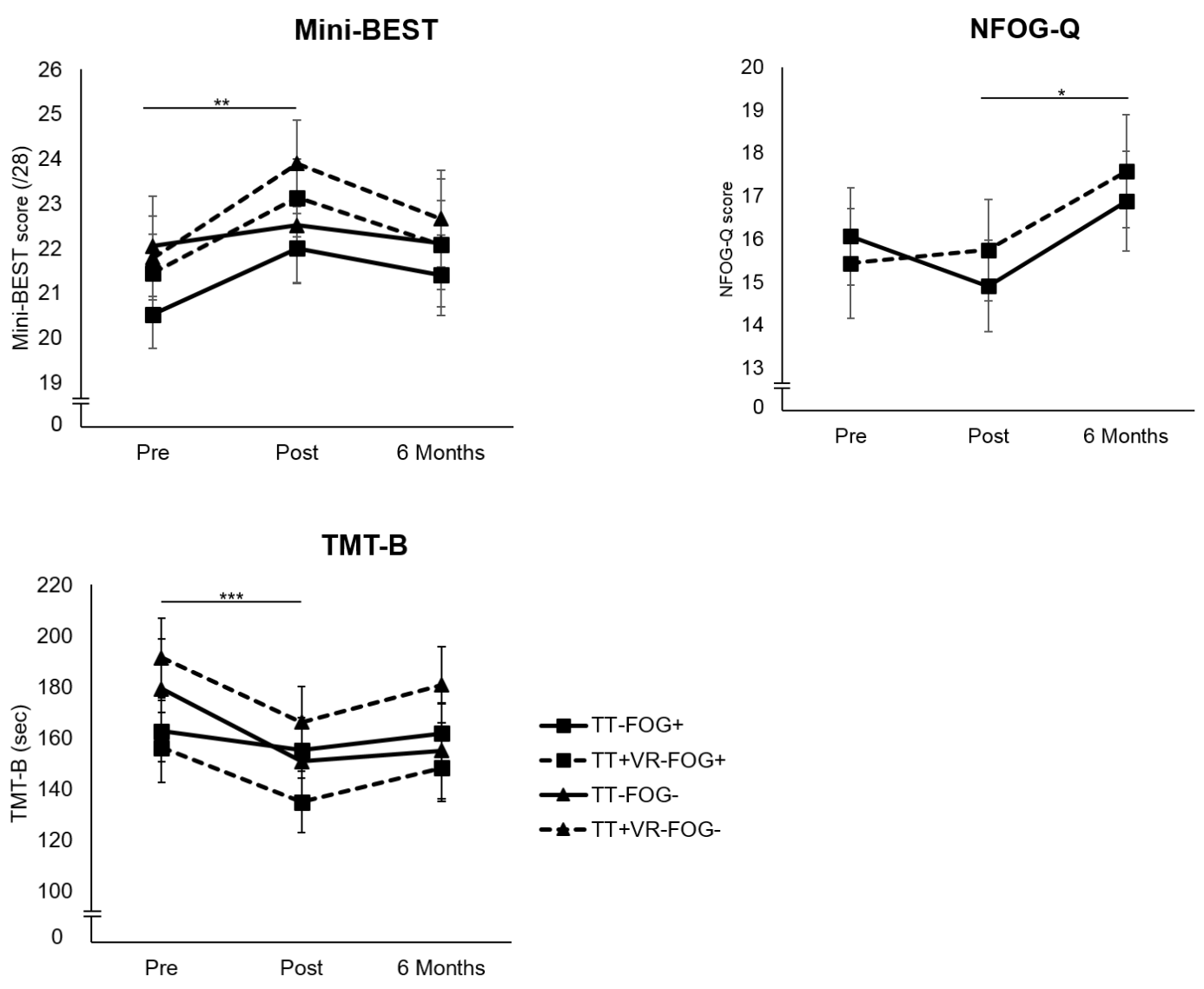
Figure 2. Training effects on falls in both PD groups with and without FOG and training arms with and without VR pre- and post-training and at 6 months follow-up. An Intervention*Time interaction $(p=0.027)$ showed a significantly greater reduction of falls in the TT+VR group in both FOG+ and FOG-. Fall incidence rate was lower in the VR group than in TT alone (IRR 0.35, 95\% CI 0.21-0.57). Fall rates were generally higher in FOG+ compared to FOG$(\mathrm{p}<0.001) .{ }^{*} \mathrm{p}<0.05, * * \mathrm{p}<0.01, * * * \mathrm{p}<0.001$.
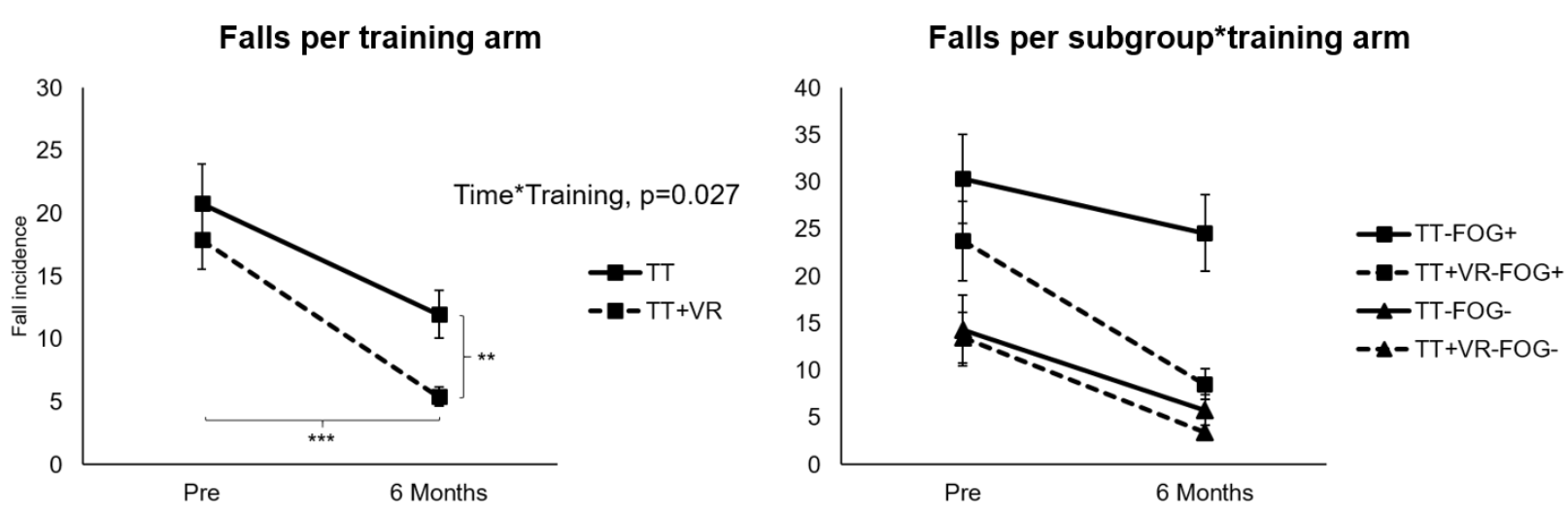


\section{Supplementary materials}

Table 1. Groups characteristics within FOG-groups between training-arms

\begin{tabular}{lccr|ccc}
\multicolumn{4}{c}{ FOG- } & \multicolumn{3}{c}{ FOG+ } \\
& $\mathbf{T T}$ & $\mathbf{T T + V R}$ & $\boldsymbol{p}$-value & $\begin{array}{c}\mathrm{TT} \\
\mathbf{n = 4 3}\end{array}$ & $\begin{array}{c}\text { TT+VR } \\
\mathbf{n = 3 4}\end{array}$ & $\boldsymbol{p}$-value \\
\hline Age & $71.31 \pm 6.2$ & $71.86 \pm 6.5$ & 0.787 & $70.70 \pm 6.1$ & $70.41 \pm 6.1$ & 0.838 \\
Education & $13.38 \pm 4.9$ & $13.43 \pm 4.3$ & 0.970 & $13.21 \pm 4.3$ & $13.24 \pm 4.3$ & 0.983 \\
Gender (m/f) & $8 / 8$ & $17 / 11$ & 0.490 & $29 / 14$ & $20 / 14$ & 0.435 \\
Disease duration (yrs) & $5.12 \pm 4.5$ & $8.39 \pm 5.2$ & $\mathbf{0 . 0 4 4}$ & $11.09 \pm 7.4$ & $9.59 \pm 5.7$ & 0.332 \\
H\&Y & $2.28 \pm 0.5$ & $2.20 \pm 0.4$ & 0.516 & $2.57 \pm 0.5$ & $2.60 \pm 0.4$ & 0.753 \\
MMSE & $28.19 \pm 1.6$ & $27.46 \pm 1.6$ & 0.149 & $28.40 \pm 1.5$ & $28.00 \pm 1.8$ & 0.288 \\
FOGQ & $/$ & $/$ & n.a. & $16.05 \pm 7.0$ & $15.65 \pm 6.8$ & 0.802 \\
MoCA & $24.56 \pm 3.6$ & $23.89 \pm 4.8$ & 0.635 & $24.16 \pm 3.5$ & $23.82 \pm 4.3$ & 0.701 \\
UPDRS-III & $26.00 \pm 16.0$ & $26.18 \pm 9.7$ & 0.968 & $30.63 \pm 11.9$ & $33.35 \pm 14.7$ & 0.372 \\
Falls & $18.06 \pm 51.9$ & $15.14 \pm 56.6$ & 0.866 & $29.56 \pm 74.8$ & $22.15 \pm 51.1$ & 0.623 \\
Mini-BEST & $28.88 \pm 6.4$ & $55.57 \pm 5.8$ & 0.873 & $20.30 \pm 5.9$ & $20.62 \pm 5.7$ & 0.813 \\
FES-I & $31.44 \pm 11.3$ & $30.19 \pm 9.8$ & 0.704 & $38.32 \pm 10.9$ & $34.79 \pm 12.8$ & 0.205 \\
\hline
\end{tabular}

Abbreviations: FES-I: Falls Efficacy Scale-International; FOG+: patients with freezing of gait; FOG-: patients without freezing of gait; H\&Y: Hoehn \& Yahr; Mini-BEST: Mini-Balance Evaluation System Test; MMSE: Mini-Mental State Examination; MoCA: Montreal Cognitive Assessment; NFOG-Q: New Freezing of Gait Questionnaire; TT: Treadmill training; TT+VR: Treadmill training plus virtual reality; UPDRS-III: Unified Parkinson's Disease Rating Scale motor part (III). 